\title{
A New Approach for Evaluation of the Hubble and the Deceleration Parameters
}

\author{
Mohammed K. Zeki Nazzeha A. Daod Aziz J. Abdel-Kadder \\ Department of Physics \\ College of Science \\ Mosul University
}

(Received 10 / 2 / 2010 ; Accepted 13 / 7 / 2010 )

\begin{abstract}
In this work we studied the dependence of the Cosmological parameters (the Hubble $\mathrm{H}(\mathrm{t})$ and deceleration $\mathrm{q}(\mathrm{t})$ parameters) on the red shift through the scale factor $\mathrm{R}(\mathrm{t})$, so we obtained two relations for the parameters. Assuming the scale factor as a function of time to the power $\alpha$ and $\alpha(t)$, we found other sets for the parameters $H(t)$ and $q(t)$. The derived relations for $\mathrm{H}(\mathrm{t})$ and $\mathrm{q}(\mathrm{t})$ coincides with the well-known relations for the parameters.
\end{abstract}

Keywords: Cosmology, Variation of Hubble's and deceleration parameters.

\section{مدلى جيد لاحتسلب معاملي هلالل والتبللؤ}

\begin{abstract}
الملغص

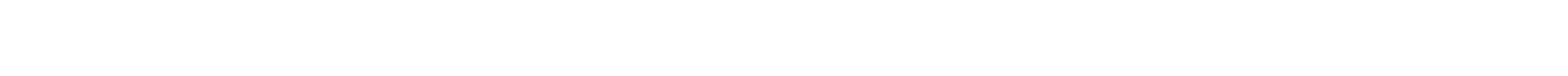
( معلطل هالب H(t) ومعلمل التبطؤ q(t) وذلك أولاً بالاعتماد على الزحزحة الحمراء من خلل علمل

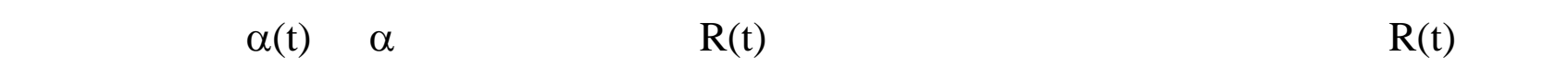

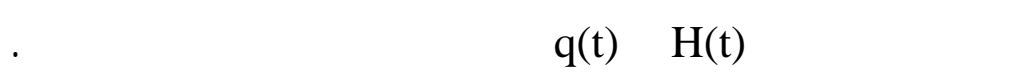

\section{INTRODUCTION}

Einstein formulated the general theory of relativity according to the static behavior of the universe. If the universe has been disturbed slightly, it might either expand to infinity with an asymptotic behavior of de-Sitter, or it might collapse according to Friedman-models (Narlikar, 1979).

Most of the model of the universe were constructed according to the interpretation of the red shift $(\mathrm{Z})$ as a function of scale factor $\mathrm{R}(\mathrm{t})$. The red shift has been explained by the hypothesis of continuous variation of the scale factor, therefore the definition of the Hubble and deceleration parameters depend on the variation of scale factor with respect to time (Weinberg, 1972). 
Solution of the field equations may be generated by applying a law of variation for Hubble's parameter, which was proposed by Berman (1983). This assumption with its simplest case, the Hubble parameter yields a constant value for deceleration parameter. This can be found in literature like, Einstein theory, Brans-Dick theory and inflationary models.

Observations (Knop et al., 2003 ; Riess et al., 2004) of type Ia supernovae (SNI) allow to probe the expansion history of the universe. Observation of the universe is accelerating, which means that the cosmological models should have variable cosmological term and deceleration parameter. (Debnath and Paul, 2005; Pradhan et al., 2006),

The key point of our work is to derive the Hubble $(\mathrm{H})$, and the deceleration (q) parameters using two different approaches. In the first approach we used the relation of scale factor as a function of red shift $\left[\frac{\mathrm{R}}{\mathrm{R}_{\mathrm{o}}}=(1+\mathrm{Z})^{-1}\right]$ to derive the Hubble and the deceleration parameters in terms of red shift while in the second approach we used the relation $\left[\frac{\mathrm{R}}{\mathrm{R}_{\mathrm{o}}}=\left(\frac{\mathrm{t}}{\mathrm{t}_{\mathrm{o}}}\right)^{\alpha}\right]$, one by considering $\alpha^{\prime}$ s is a curvature dependence and the other is a time dependence, so we got two sets of equations for the Hubble and the deceleration parameters.

\section{THEORY}

The Hubble parameter $\mathrm{H}(\mathrm{t})$ and the deceleration parameter $\mathrm{q}(\mathrm{t})$ is simply defined by (Weinberg, 1972).

$$
\begin{aligned}
& H=H(t)=\frac{\dot{R}}{R} \\
& q=q(t)=-\frac{\ddot{R}}{\dot{R}} \frac{R}{\dot{R}}
\end{aligned}
$$

Where both $\mathrm{H}$ and $\mathrm{q}$ are functions of time and $\mathrm{R}$ is the scale factor of the universe at time $\mathrm{t}(\mathrm{R}=\mathrm{R}(\mathrm{t}))$.

Starting from the precise definition of the red shift (Z) (Weinberg, 1972).

$$
\mathrm{Z}=\frac{\lambda_{0}-\lambda}{\lambda}=\frac{\nu}{v_{0}}-1
$$

Where, $\lambda_{0}$ and $\lambda$ are observed and source wavelengths with their associated frequencies $v_{0}$ and $v$ respectively, and according to Weinberg 1972, we have:

$$
\frac{v_{0}}{v}=\frac{t}{t_{0}}=\frac{R}{R_{0}}
$$

Where, $R_{0}$ is the scale factor at the present time $t_{0}$.

Combining equations (3) and (4), then

$$
\mathrm{R}=\mathrm{R}_{\mathrm{o}}(1+\mathrm{Z})^{-1}
$$


First approach: we find the Hubble $(\mathrm{H})$ and deceleration $(\mathrm{q})$ parameters, by differentiating equation (5) with respect to time we get:

$$
\dot{\mathrm{R}}=-\mathrm{R}_{\mathrm{o}}(1+\mathrm{Z})^{-2} \dot{\mathrm{Z}}
$$

dividing equation (6) by equation (5) and substituting in equation (1), then:

$$
\mathrm{H}=-\dot{\mathrm{Z}}(1+\mathrm{Z})^{-1}
$$

Equation (7) is the Hubble parameter in terms of the red shift (Z).

Similarly, to find the deceleration parameter (q), we differentiate equation (6) with respect to time, and substitute in equation (2) we get.

$$
q=\frac{\ddot{Z}}{\cdot \overrightarrow{2}}(1+Z)-2
$$

To eliminate $\ddot{Z}$ in equation (8), we differentiate equation (7) with respect to time:

$$
\begin{aligned}
& \dot{H}=-\left[\ddot{Z}(1+Z)^{-1}-\stackrel{2}{Z}(1+Z)^{-2}\right] \\
& \text { or } \\
& \ddot{Z}=\stackrel{\bullet}{Z}(1+Z)^{-1}-\dot{H}(1+Z)
\end{aligned}
$$

Now combining equations (7), (8) and (9), we get:

$$
\mathrm{q}=-\left[1+\frac{\dot{\mathrm{H}}}{\mathrm{H}^{2}}\right]
$$

Equation (10) is a well-known equation in cosmology which verify that our work is correct. Second approach: We reformulate equation (4) as:

$$
R=R_{o}\left(\frac{t}{t_{o}}\right)^{\alpha}
$$

where $\alpha$ is a constant and takes different values $(0.5769,0.6921,0.9997)$ depending on the type of curvature $(\mathbf{K}=1,0,-1)$ respectively (Weinberg, 1972; Olive and Peacock, 2002,). Hubble parameter $(\mathrm{H})$ can be found by differentiating equation (11) with respect to time, then:

$$
\mathrm{H}=\frac{\dot{\mathrm{R}}}{\mathrm{R}}=\frac{\alpha}{\mathrm{t}}
$$

So $\alpha=\mathbf{H} \mathbf{t}$ verifying the correctness of our assumption.

Now differentiating the logarithmic form of equation (12) we get: 


$$
\frac{\ddot{\mathrm{R}}}{\dot{\mathrm{R}}}=\frac{\dot{\mathrm{R}}}{\mathrm{R}}-\frac{1}{\mathrm{t}}=\mathrm{H}-\frac{1}{\mathrm{t}}
$$

Then combining equation (2), (12) and (13), we get:

$$
q=-\left(1-\frac{1}{\alpha}\right)
$$

At , $\alpha \approx(0.99997,0.5769,0.6921)$, then q values are $\left(<\frac{1}{2},>\frac{1}{2},=\frac{1}{2}\right)$ respectively, which is exactly as given by Weinberg 1972 .

To verify that (14) is the same form as equation (10), we differentiate equation (12) with respect to time and dividing by $\mathrm{H}^{2}$ then:

$$
\frac{\dot{\mathrm{H}}}{\mathrm{H}^{2}}=\frac{-\frac{\alpha}{\mathrm{t}^{2}}}{\frac{\alpha^{2}}{\mathrm{t}^{2}}}=-\frac{1}{\alpha}
$$

Therefore equation (14) reads:

$$
q=-\left[1+\frac{\dot{H}}{H^{2}}\right]
$$

This verify that equation (16), is consistent with equation (10), that was derived from the first approach.

Now equation (11) can be written in a more general form, assuming that $\alpha$ is no longer a constant but it is time dependence (Similar to Mansonri et al., 1999; Troitskii, 1987), hence:

$$
\frac{\mathrm{R}}{\mathrm{R}_{\mathrm{o}}}=\left(\frac{\mathrm{t}}{\mathrm{t}_{\mathrm{o}}}\right)^{\alpha(\mathrm{t})}
$$

Now again differentiating the logarithmic form of equation (17) with respect to time:

$$
\begin{aligned}
& \frac{\dot{\mathrm{R}}}{\mathrm{R}}=\frac{\alpha(\mathrm{t})}{\mathrm{t}}+\dot{\alpha}(\mathrm{t}) \ln \left(\frac{\mathrm{t}}{\mathrm{t}_{\mathrm{o}}}\right) \\
& \text { or } \\
& \frac{\mathrm{t}}{\alpha(\mathrm{t})} \frac{\dot{\mathrm{R}}}{\mathrm{R}}=1+\frac{\dot{\alpha}(\mathrm{t})}{\alpha(\mathrm{t})} \mathrm{t} \quad \ln \left(\frac{\mathrm{t}}{\mathrm{t}_{\mathrm{o}}}\right)
\end{aligned}
$$


Mathematically:

$$
\begin{aligned}
& \frac{\dot{\alpha}(\mathrm{t})}{\alpha(\mathrm{t})} \text { is much less than } \mathrm{t} \quad \mathrm{ln}\left(\frac{\mathrm{t}}{\mathrm{t}_{\mathrm{o}}}\right) \text { and this implies: } \\
& \frac{\mathrm{t}}{\alpha(\mathrm{t})} \frac{\dot{\mathrm{R}}}{\mathrm{R}}=1 \\
& \mathrm{H}=\frac{\alpha(\mathrm{t})}{\mathrm{t}}
\end{aligned}
$$
(12).

Equation (20 b) is the general form of the Hubble parameter which look likes equation

Deceleration parameter $(q)$ can be found by differentiating the logarithmic form of equation (20a) with respect to time, then:

$$
\frac{\ddot{\mathrm{R}}}{\dot{\mathrm{R}}}=\frac{\dot{\mathrm{R}}}{\mathrm{R}}+\frac{\dot{\alpha}(\mathrm{t})}{\alpha(\mathrm{t})}-\frac{1}{\mathrm{t}}
$$

Now $\dot{\alpha}(\mathrm{t})$ can be found by differentiating equation (20b) with respect to time:

$\dot{\alpha}(\mathrm{t})=\dot{\mathrm{H}} \mathrm{t}+\mathrm{H}$

Combining equation (1), (2), (21) and (22) we get:

$$
\mathrm{q}=-\left(1+\frac{\dot{\mathrm{H}}}{\mathrm{H}^{2}}\right)
$$

Equation (23) is also consistent with equation (10), which means that our approximation gives accurate result.

\section{CONCLUSION}

In this work we obtained different sets of equations defining the Hubble $(\mathrm{H})$ and the deceleration (q) parameters using two different approaches.

In the first approach, we started our derivation with the variation of the scale factor equation (5) with respect to time, which leads us to a new form of the Hubble and deceleration parameters as a function of red shift (equation $7 \& 8$ ). From these two equations we obtained the well-known relation (10) for the definition of the deceleration parameter, which is commonly used in most of the text books. This emphasize the reality of our work.

In the second approach we used two assumptions, one by starting our derivation through the variation of the scale factor equation (11) considering $\alpha$ as a curvature dependence, which leads us to equation (12) and (14). Again simplifying equation (14) gives the same form of the equation (10).

The other assumption considering $\alpha$ in equation (17) as a function of time, we got two relations (20b, 23), equation (20b) defining the general form of the Hubble's parameter $\mathrm{H}$ with $\alpha$ as a time dependence, which look likes equation (12). Whereas equation (23) is also consistent with equation (10), this means that our approximation is correct. Also we 
conclude that $\frac{\dot{\mathrm{R}}}{\mathrm{R}}=\frac{\dot{\mathrm{G}}}{\mathrm{G}}=\frac{\dot{\mathrm{c}}}{\mathrm{c}}=\mathrm{H}(\mathrm{t})$ where $\mathrm{R}, \mathrm{G}$ and c are the scale factor, gravitational constant and the speed of light respectively (Troitskii, 1987; Guenther et al., 1997; Mansouri et al., 1999).

Finally we want to emphasis that the derived parameter $\mathrm{q}$ is still describing the constant deceleration of the universe. In future work one can study the variation of both $\mathrm{H}$ and q parameters with time, in which q parameter will be no longer a constant, but will acts as an accelerating parameter of the universe (Debnath and Paul, 2005; Pradhan et al., 2006).

\section{REFERENCES}

Berman, M. S. (1983). A special law of variation of hubble's parameter. Nuov Cimento B, 74B, 182-186.

Debnath, P. S. ; Paul, B. (2005). Cosmological models with variable gravitational and cosmological constants in $\mathrm{R}^{2}$ gravity. arXiv: gr-qc/0508031, V2.

Guenther, D. B.; Krauss, L. M.; Demarque, P. (1997). Testing the constancy of the gravitational constant using helioseismology. Astrophysical J. , 498, 871-876.

Knop, R. A.; Aldering, G.; A,anullah, R.; Asiter, P.; Blanc, G.; Burns, M. S.; Conley, A.; Deustua, S. E.; Doi, M.; Ellis, R.; Fabbro, S.; Folatelli,G.; Fruchter, A. S.; Garavini, G.; Garmond, s.; Garton, K.; Gibbons, R.; Goldhaber, G.; Goobar, A.; Groom, D. E.; Hardin, D.; Hook, i.; Howell, D. A.; Kim, A. G.; Lee, B. C.; Lidman, C.; Mendez, J.; Nobili, S.; Nugent, P. E.; Pain, R.; Panagia, N.; Pennypacker, C. R.; Perlmutter, S.; Quimby, R.; Raux, J.; Regnault, N.; Ruiz-Lapuente, p.; Sainton, G.; Schaefer, B.; Schahmaneche, K.; Smith, E.; Spadafora, A. L.; Stanishev, V.; Sullivan, M.; Walton, N. A.; Wang, L.; Wood-Vasey, W. M.; Yasuda, N. (2003). New constraints on $\boldsymbol{\Omega}_{\boldsymbol{M}}, \boldsymbol{\Omega}_{\wedge}$, and $\boldsymbol{w}$ from an independent set of 11 high-redshift supernovae observed with the Hubble Space Telescope,Astrophys. J., 598, 102-137.

Mansouri, R.; Nasseri F.; Khoriami M. (1999). Effect time variation of G in a model universe with variable space dimension. Physics Letter A, 259, 194-200.

Narlikar, J. V. (1979). "General Relativity and Cosmology", the Macmillan Press Ltd., pp.188-203.

Olive, K. A.; Peacock J. A. (2002). Big bang cosmology. Physical Review D66:010001152-161.

Pradhan, A.; Shahi J. P. ; Singh, C. B.(2006). Cosmological models of universe with variable deceleration parameter in Lyra's manifold. Brazilian J. Physics, 36 (4A).

Riess, A. G.; Strolger, L-G.; Tonry, J.; Casertano, S.; Ferguson, H. C.; Mobasher, B.; Challis, P.; Filippenko, A. V.; Jha, S.; Li, W.; Chornock, R.; Kirshner, R. P.; Leibundgut, B.; Dickinson, M.; Livio, M.; Giavalisco, M.; Steidel, C. C.; Benitez, T.; Tsvetanov, Z. (2004). Type la supernova discoveries at $\mathrm{z}>1$ from the Hubble Space Telescope: evidence for past deceleration and constraints on dark energy evolution. Astrophys. J. 607, 665.

Troitskii, V. S. (1987). Physical constants and evaluation of the universe. Astrophysics and Space Science. 139, 389-411.

Weinberg, S. (1972). "Gravitation and Cosmology". John Wily and Sons. Inc., New York, pp.407-468. 